\title{
Tricyclic 2-Aminopyridinium Derivatives with Aggregation-Induced Emission for Concomitant Bacterial Discrimination and Inhibition
}

\author{
Bingnan Wang, ${ }^{\dagger}$ Haozhong Wu, ${ }^{\dagger}$ Rong Hu, ${ }^{\dagger}$ Xiaolin Liu, Zhiyang Liu, ${ }^{\ddagger}$ Zhiming Wang, ${ }^{\dagger}$ Anjun Qin ${ }^{*},{ }^{\dagger}$ \\ and Ben Zhong Tang,",
}

'State Key Laboratory of Luminescent Materials and Devices, Guangdong Provincial Key Laboratory of Luminescence from Molecular Aggregates, SCUT-HKUST Joint Research Institute, Center for Aggregation-Induced Emission, South China University of Technology (SCUT), Guangzhou 510640, China

${ }^{\ddagger}$ Department of Chemistry, Hong Kong Branch of Chinese National Engineering Research Centre for Tissue Restoration and Reconstruction, Institute for Advanced Study, and Department of Chemical and Biological Engineering, The Hong Kong University of Science \& Technology (HKUST), Clear Water Bay, Kowloon, Hong Kong, China

\begin{abstract}
The development of new ionic compounds with aggregation-induced emission (AIE) has been widely noticed. They can not only effectively solve the problem of aggregation-caused quenching (ACQ) encountered in conventional fluorescent dyes, but also exhibit promising applications in biological imaging, diagnosis and therapy, etc. However, such AIE system should be further developed. In this work, a series of novel cationic AIE luminogens (AIEgens) of tricyclic 2-aminopyridinium derivatives with seven-membered rings were designed and synthesized via a simple multi-component reaction, which exhibit the ability to specifically stain gram-positive bacteria. Meanwhile, they also possess excellent bacteriostatic ability for $S$. aureus in liquid medium and solid agar plates, of which the minimum inhibitory concentration (MIC) value is between 4 and 8 $\mu \mathrm{g} / \mathrm{mL}$. In addition, these molecules showed mitochondria-targeting capability in hydrophilic AIEgens and specific staining lysosomes in lipophilic AIEgens by wash-free process in living cells. These AIEgens with bacteriostatic activity hold great promise for distinguishing bacteria and inhibiting bacterial infection in clinical trial.
\end{abstract}

\section{Introduction}

Bacterial infections usually cause serious illnesses such as dermatosis, pneumonia and septic arthritis, which have increasingly raised and threatened the public health. ${ }^{1}$ Reliable and rapid diagnosis and effective therapy of bacterial infection are two key steps in clinical research. ${ }^{2}$ The drugresistant bacteria have been generated due to the overuse of antibiotics in therapeutic process. For example, Grampositive methicillin-resistant $S$. aureus (MRSA) is resistant to multiple antibiotics and causes common infectious diseases in hospital.3,4 Therefore, it is of great significance for the research of pharmacology and biomedicine to develop new structures of bacteriostatic activity.

Diagnosis of bacterial infection is the precondition of effective and precise treatment. At present, Gram-staining method is a traditional way for distinguishing Gram-positive bacteria from Gram-negative bacteria, while the Gramstaining process is extremely complicated and in situ detection of living bacteria is impossible. Other generally adopted methods for bacteria identification include gene microarray, ${ }^{5}$ immunological analysis, ${ }^{6}$ polymerase chain reaction, ${ }^{7}$ and biochemical sensing, ${ }^{8}$ which are time-consuming, complex in operation and request sophisticated instruments. To overcome these shortcomings, new methods to achieve fast and accurate bacterial identification are essential.

Recently, fluorescence technology has been rapidly developed and applied in bacterial identification, ${ }^{9-17}$ because of its remarkable advantages of simplicity and superior sensitivity, etc. ${ }^{18}$ However, the majority of traditional fluorescent materials are lipophilic and tend to aggregate in the biological aqueous environment, and thus exhibit aggregation-caused quenching (ACQ) effect, where fluorescence intensity is quenched or weakened in the aggregate state or at high concentration. The ACQ effect confines operating levels at lower concentrations and limits long-term tracking due to labeling degrees, which restrict their applications in biological imaging, accurate diagnosis and photodynamic therapy. ${ }^{19,20}$

Aggregation-induced emission (AIE), an exactly opposed phenomenon to the conventional ACQ effect, was proposed by our group in 2001, which shows stronger emission of a luminogen in the aggregate state than that in solution. ${ }^{21} \mathrm{Up}$ to now, multifarious AIE luminogens (AIEgens) have been synthesized under the guidance of the AIE mechanism of restriction of intramolecular motion (RIM), which includes restriction of intramolecular motion (RIR), and restriction of intramolecular vibration (RIV). It is worth mentioning that cationic AIEgens, as a main component, are outstandingly developed in bioimaging, biosensing, biological diagnosis and treatment, ${ }^{21-29}$ resulting from their admirable characteristics of high quantum yields in aggregate state, good water solubility, large Stokes shift, turn-on feature in bioassay, high signal-to-noise ratio, and admirable photostability. ${ }^{30-38}$ 
<smiles></smiles>

BMTAPC-5<smiles></smiles>

BMTAPC-7<smiles></smiles>

BMTAP-7

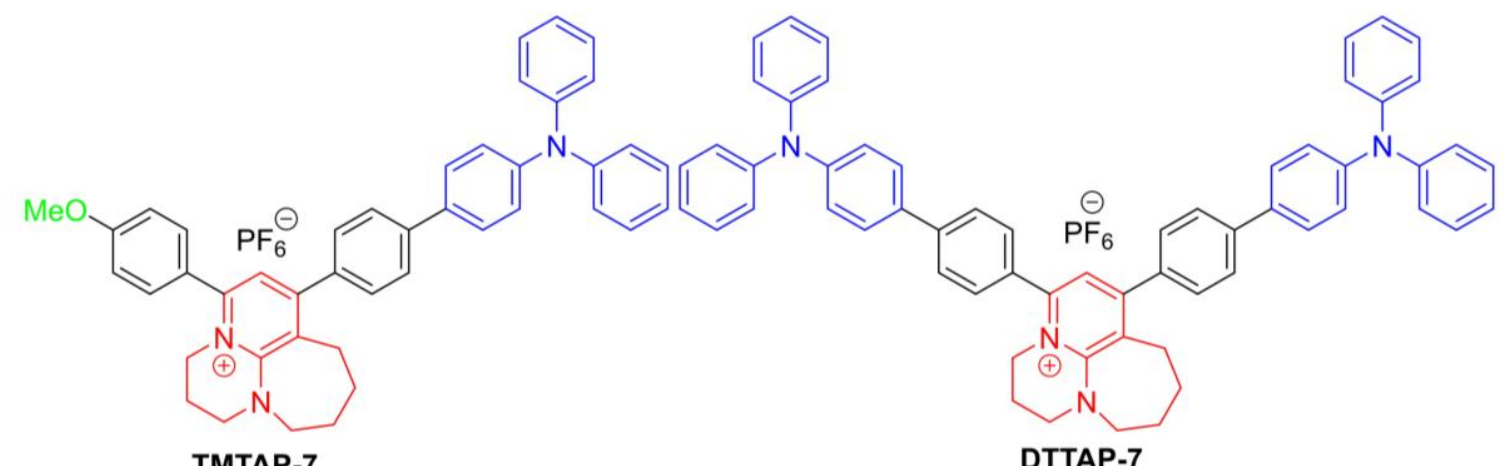

TMTAP-7
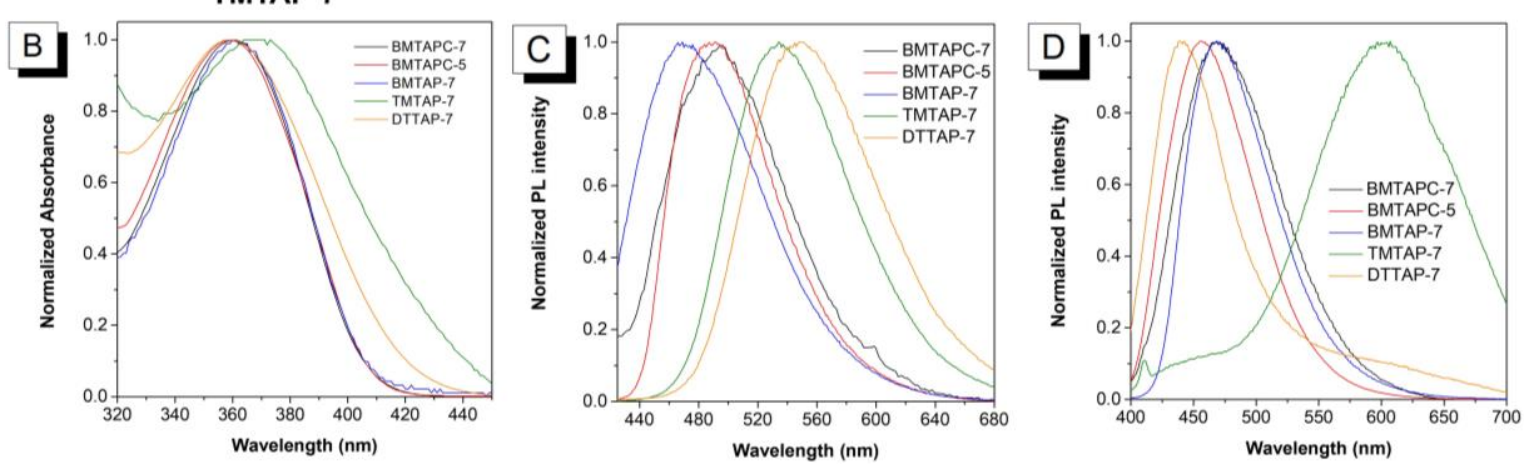

Figure 1. (A) Molecular structures of the designed materials. (B) Normalized absorption spectra of BMTAPC-5, BMTAPC-7, BMTAP7, TMTAP-7 and DTTAP-7 dissolved in DCM or THF. (C) Normalized PL spectra of five molecules in solid. (D) Normalized PL spectra BMTAPC-5, BMTAPC-7, BMTAP-7, TMTAP-7 and DTTAP-7 in DCM or THF.

However, there are some potential issues that need to be addressed. In terms of water-soluble elements, cationic AIEgens are mainly pyridine, triphenyl phosphorous and quaternary ammonium salts, ${ }^{39-43}$ which are uneasy to separate by column chromatography and particularly modify their structures. Notably, most of AIEgens are synthesized according to mechanism of RIR, while few functional groups were reported by RIV as a mechanism. For applications of integrated diagnosis and treatment, bacterial discrimination has been found recently, ${ }^{44,45}$ but the AIEgens with the capacity of discrimination and pharmacologic activity for bacteria are rarely reported.

Based on the above issues, we synthesized a series of cationic tricyclic 2-aminopyridinium derivatives with 1,8-diazabicyclo[5.4.0] undec-7-ene (DBU), an organic base commonly used in organic chemistry, which is a functional group featuring the mechanism of RIV, ${ }^{46}$ and discussed their photophysical properties, theoretical calculations, organelle specific imaging, ability of bacterial discrimination and inhibition. Meanwhile, the bacteriostatic mechanism was further explored by control experiment to find possible targets. This study not only provides novel cationic AIEgens with the capacity of discrimination and inhibition for bacteria, but also promotes the development of bacteriostatic materials.

\section{Results and Discussion}

\section{Design and synthesis of molecular structures}

Compounds BMTAPC-5, BMTAPC-7, BMTAP-7, TMTAP-7 and DTTAP-7, whose structures are shown in Figure 1A, were designed and synthesized by simple organic reactions as reported in literature. ${ }^{47}$ Experimental procedures and structural characterization data are provided in the Supporting Information.

These compounds are composed of electron acceptor of tricyclic 2-aminopyridinium moiety, electron donor of triphenylamine (TPA), bromine, $\pi$-bridge and electron donor of benzene ring, and/or electron donor of methoxyl group. As a result, they demonstrate electron donating-accepting interactions and change of conjugated length. For BMTAPC5 and BMTAPC-7, when the five- and seven membered-ring are introduced into the two molecules, respectively, the luminescent properties of them are dramatically different in solutions and solid states. ${ }^{46}$ Moreover, the positively charged tricyclic 2-aminopyridinium moiety not only act as electron acceptor, but also endow these compounds to potentially apply in imaging of negatively charged bacteria and antibacterial activity. ${ }^{48}$ Meanwhile, for BMTAP-7, TMTAP-7 and DTTAP-7, triphenylamine segments are gradually added to the molecular structures as an 
Table 1. Photophysical properties of five compounds a

\begin{tabular}{lccccccc}
\hline & $\lambda_{\text {abs }}[\mathrm{nm}]$ & \multicolumn{2}{c}{$\lambda_{\mathrm{em}}[\mathrm{nm}]$} & \multicolumn{2}{c}{$\Phi_{\mathrm{F}}[\%]$} & \multicolumn{2}{c}{$\tau(\mathrm{ns})$} \\
& soln & soln & solid film & soln & solid film & soln & solid film \\
\hline BMTAPC-7 & 361 & 471 & 494 & 3.5 & 21.7 & 0.7 & 3.0 \\
BMTAPC-5 & 359 & 457 & 492 & 31.3 & 1.6 & 2.4 & 1.3 \\
BMTAP-7 & 361 & 469 & 469 & 3.4 & 28.5 & 0.8 & 4.1 \\
TMTAP-7 & 365 & $450 / 607$ & 534 & 8.3 & 16.5 & 0.9 & 16.4 \\
DTTAP-7 & 360 & $450 / 603$ & 550 & 5.0 & 10.2 & 2.0 & 13.6 \\
\hline
\end{tabular}

a Photophysical properties of BMTAPC-7 and BMTAPC-5 were tested in DCM solution, which for BMTAP-7, TMTAP-7 and DTTAP-7 were tested in THF solution.

electron-donating group, which affect their luminescent properties and hydrophilicity. Through comparison of the photophysical property of BMTAP-7, TMTAP-7 and DTTAP7 , the property-structure relationship could be explored in depth and it would be attractive to investigate the effects of hydrophilic and hydrophobic interactions on bacterial imaging and antibacterial activity.

\section{Photophysical properties}

After confirming their structures, the photophysical properties of five compounds were investigated. Figure 1B1D shows the UV/Vis absorption and photoluminescence (PL) spectra in their different states and Table 1 summarizes the corresponding data. The absorption peak $\left(\lambda_{\mathrm{abs}}\right)$ at $361 \mathrm{~nm}$ of BMTAPC-7 is close to that ( $\lambda_{\text {abs }}$ of $359 \mathrm{~nm}$ ) of BMTAPC-5 in dilute dichloromethane (DCM) solutions. TMTAP-7 shows a slightly red-shifted absorption peak $\left(\lambda_{\mathrm{abs}}\right.$ at $365 \mathrm{~nm}$ ) compared with those of BMTAP-7 and DTTAP-7 $\left(\lambda_{\text {abs }}\right.$ of $360 \mathrm{~nm}$ ) in dilute THF solutions (Figure 1B). From the PL spectra of compounds in solid states (Figure 1C), we can find the maximum emission peaks of BMTAPC-5 and BMTAPC-7 are at $493 \mathrm{~nm}$, while TMTAP-7 and DTTAP-7 show redder emission peaks ( $\lambda_{\mathrm{em}}$ at 534 and $550 \mathrm{~nm}$ ) than that of BMTAP-7 ( $\lambda_{\text {em }}$ of $469 \mathrm{~nm}$ ), which is caused by the addition of triphenylamine segments to increase the molecular conjugation. In solutions, these five compounds have a similar emission wavelength from 400 to $500 \mathrm{~nm}$, but TMTAP7 and DTTAP-7 exhibit longer wavelength emission peaks at about $607 \mathrm{~nm}$ (Figure 1D). The difference in their absorption and emission peaks is probably due to their different conjugation.

Subsequently, the PL spectra of BMTAPC-5 and BMTAPC7 in DCM $/ n$-hexane mixtures with different $n$-hexane fractions $\left(f_{\mathrm{h}}\right)$ were measured to study the source of the AIE property (Figure S1 and Table 1), since BMTAPC-5 and BMTAPC-7 are more soluble in DCM and could not be dissolved in $n$-hexane. When $f_{\mathrm{h}}$ is $99 \%$, the PL intensity of BMTAPC-7 enhanced sharply. However, the PL intensity of BMTAPC- 5 decreased with the increase of $f_{\mathrm{h}}$. This phenomenon is because the molecules form aggregations in high $f_{\mathrm{h}}$, which limits intramolecular seven-member ring vibration and leads to the PL enhancement. ${ }^{46}$ The absolute PL quantum yield $\left(\Phi_{\mathrm{F}}\right)$ measurement further confirms above phenomenon. BMTAPC-7 showed a lower $\Phi_{\mathrm{F}}$ value $(3.5 \%)$ in DCM solution than in solid film (21.7\%), whereas BMTAPC5 showed a higher $\Phi_{F}$ value $(31.3 \%)$ in DCM than in the solid film $(1.6 \%)$. These results demonstrate the main reason of AIE is that intramolecular seven-member ring vibration is restricted in solid or aggregate state.

Thanks to their donor- $\pi$-acceptor structures, TMTAP-7 and DTTAP-7 exhibit obvious solvatochromic effect. The PL intensity decreased with the increase of solvent polarity (Figure S2). Solvatochromic effects of TMTAP-7 and DTTAP-7 lead to a significant PL enhancement for the two molecules in low polar solutions, so $\mathrm{DCM} / n$-hexane system is not suitable for testing AIE properties of TMTAP-7 and DTTAP-7. We thus change the solvent mixture to THF/water, which could avoid the problem of PL enhancement caused by solvatochromic effect. As shown in Figure S1 and Table 1, for BMTAP-7, TMTAP-7 and DTTAP-7, their AIE effects also are activated in THF/water mixtures with high water fractions and show higher $\Phi_{\mathrm{F}}$ values in solid state than those in THF solution. Moreover, PL lifetime measurements reveal that these seven-member ring AIEgens show longer lifetime in solid state than in solution (Table 1) because the nonrediative transition is limited in solid states. ${ }^{46}$

\section{Theoretical calculations}

To better understand the distinction of the photophysical properties caused by structural modifications, BMTAP-7, TMTAP-7 and DTTAP-7 were further investigated via density functional theory (DFT) calculations. The geometries of BMTAP-7, TMTAP-7 and DTTAP-7 were optimized with B3LYP/6-31G** level (Figure 2). The highest occupied molecular orbitals (HOMOs) of these AIEgens are mainly localized at TPA moiety or 4-methoxyphenyl group, whereas their lowest unoccupied molecular orbitals (LUMOs) are distributed at the tricyclic 2-aminopyridinium or whole molecules. Moreover, the calculated HOMO-LUMO energy gaps of TMTAP-7 and DTTAP-7 are smaller than BMTAP-7, because there are stronger electron donating-accepting 


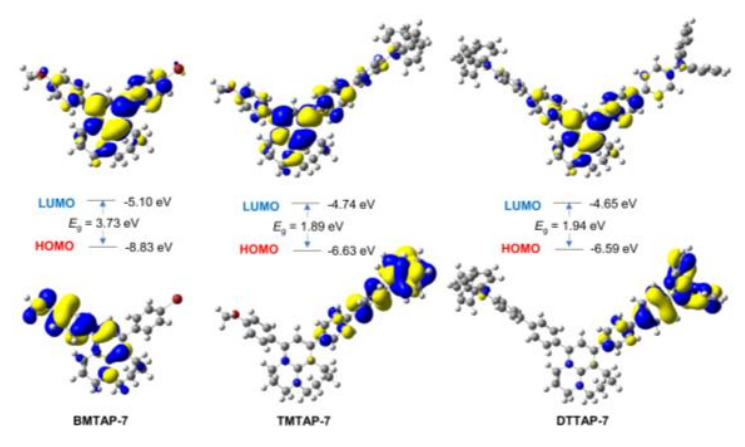

Figure 2. Molecular orbital amplitude plots of HOMO and LUMO energy levels of BMTAP-7, TMTAP-7 and DTTAP-7 in cationic state calculated at the B3LYP/6-31G** level.

interactions between TPA and tricyclic 2-aminopyridinium, which are in good accordance with the redder emission peaks.

\section{In vitro fluorescence imaging}

For bio-related applications, the biocompatibility of compounds is a key issue. We thus tested the cytotoxicity of BMTAP-7, TMTAP-7 and DTTAP-7 by standard MTT assay. The cell viabilities of these AIEgens are over $80 \%$ at concentrations below $2 \mu \mathrm{M}$ (Figures S3), exhibiting a good biocompatibility and facilitating their application in biological imaging. Indeed, after the AIEgens were added to the medium to incubate with HeLa cells for $30 \mathrm{~min}$, fluorescence signals recorded by confocal laser scanning microscopy (CLSM) were observed for BMTAP-7 and TMTAP-7 in the cytoplasm, revealing their good membrane permeability. However, obvious fluorescence was found in HeLa cells when DTTAP-7 was used after $4 \mathrm{~h}$ (Figures S4), which suggested a relatively weak ability to cross cell membranes. The reason might be that strong hydrophobicity of DTTAP-7 leads to the formation of large nanoparticles in the solution.

To confirm the intracellular location of the AIEgens, colocalization imaging experiments were performed with MitoTracker Red (MTR), a commercial mitochondria dye. The results indicated that BMTAP-7 and TMTAP-7 showed excellent overlap with MTR (Figures S4), and the Pearson's correlation coefficients were deduced to be as high as 0.91 and 0.93 because of the interaction between positively charged organic molecules and negative membrane potential of mitochondria. ${ }^{49,50}$ While the Pearson's correlation coefficient of DTTAP-7 is only 0.08 (Figures S5). DTTAP-7 might locate in the lysosome through endocytosis. DTTAP7 is similar regional distributions (Pearson's correlation coefficient of 0.63 ) in HeLa cells with commercial lysosome dye LysoTracker® Deep Red by colocalization experiments. These phenomena are closely related to the hydrophobic properties of organic molecules due to the introduction of hydrophobic TPA moiety.

\section{Selective microorganism imaging of AIEgens and expla- nation studies}

Inspired by the specific organelle staining of HeLa cells with BMTAP-7, TMTAP-7 and DTTAP-7, we further studied whether these AIEgens could be applied in selective imaging of microorganism. First, we chose S. aureus (Gram-positive bacteria) and $E$. coli (Gram-negative bacteria) as the bacteria models. As illustrated in Figure 3, after incubating

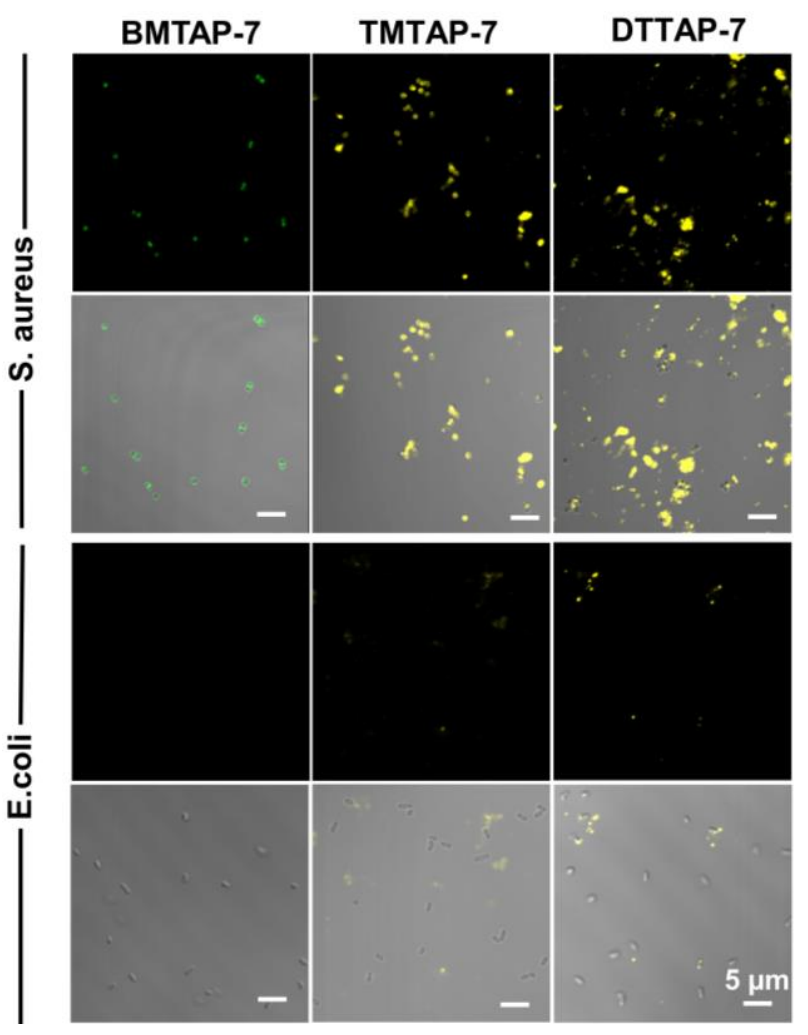

Figure 3. Fluorescence images of bacteria stained with BMTAP-7, TMTAP-7 and DTTAP-7 for $20 \mathrm{~min}$.

with S. aureus in PBS for $20 \mathrm{~min}$, the AIEgens showed excellent staining ability even without the washing process. Hydrophilic BMTAP-7 could be uniformly stained every bacterium, showing unexceptionable image effect contrast with the background. For TMTAP-7 and DTTAP-7, with the increase of their hydrophobicity, their staining ability toward $S$. aureus decreased obviously. As a sharp contrast, we found it is quite difficult for these AIEgens to stain E. coli under the same experimental conditions probably because of their multi-layer outer membrane structures. These results reveal that BMTAP-7, TMTAP-7 and DTTAP-7 could be used to distinguish between $S$. aureus and $E$. coli.

It has been confirmed that outside surfaces of Gram-positive and Gram-negative bacteria were negatively charged. ${ }^{44}$ Electrostatic adsorption is the main reason for the interaction between cationic AIEgens and bacteria, but it is not the key issue for the selective imaging behavior. We proposed that this behavior might relate to the bacterial outer membrane structures and permeability. Compared with the complex multilayer membrane structures of Gram-negative bacteria, ${ }^{45}$ the thick peptidoglycan and loose layer membrane of Gram-positive bacteria make the cationic AIEgens more easily enter. To verify this hypothesis, BMTAP-7 was used to stain dead and live E. coli and P. aeruginosa because the membrane of dead bacteria is more permeable than that of live ones. The results showed that live bacteria could not be stained (Figures 3 and S6), whereas the dead bacteria were all lit up (Figure 4A and 4B). Next, to verify the effect of specific discrimination for different microorganisms. BMTAP-7 was used to stain the dead and live fungus of $S$. cerevisiae. And the results showed that the imaging phenomena 


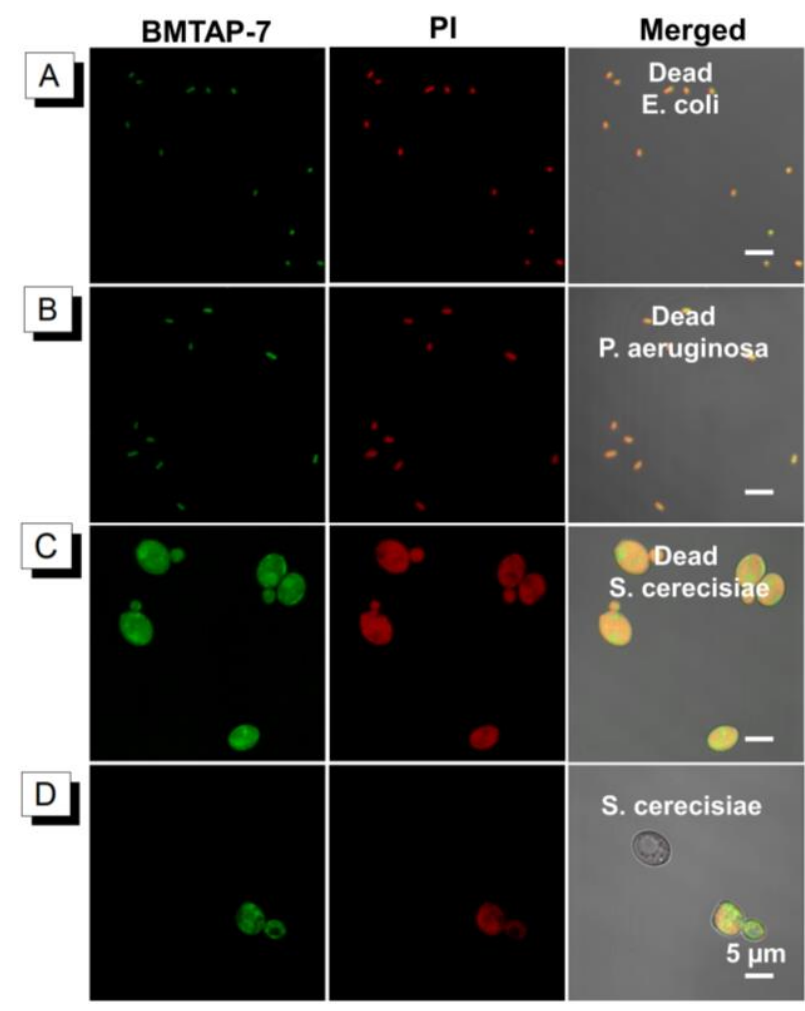

Figure 4. Fluorescence and merged images of A) dead E.coli B) dead P. aeruginosa C) dead S. cerecisiae D) S. cerecisiae incubated with BMTAP-7 and PI for 20 min.
(Figures 4C and S6) were similar to those of gram-negative bacteria. Notably, we also found that BMTAP-7 could distinguish $S$. cerevisiae in dead and live states by co-staining experiment with propidium iodide (PI). We thus conclude that the differences in the membrane structures of microorganisms lead to selective fluorescence imaging for these AIEgens.

\section{Bacteriostatic experiment and mechanism study}

Thanks to the unique structure of tricyclic 2-aminopyridinium derivatives and the ability of specific discrimination for Gram-positive bacteria, we further studied their bacteriostatic activity. First, the growth curves of bacteria were tested at various concentrations in the presence of BMTAP-7, TMTAP-7 and DTTAP-7 (Figure S7). The results showed that these AIEgens possess a significant inhibitory effect on $S$. aureus, but not on E. coli. Compared with DTTAP7, BMTAP-7 and TMTAP-7 showed effective bacteriostatic effect for $S$. aureus, possibly ascribed to their stronger binding ability as revealed by the fluorescence imaging results.

Second, the bacteriostatic experiments of these AIEgens were performed in solid agar medium. DTTAP-7, BMTAP-7 and TMTAP-7 were added into the melted agar medium to make agar plates with different concentrations from 0 to 16 $\mu \mathrm{g} / \mathrm{mL}$, respectively. After the agar plates were coated with bacteria for 24 hours, it was found that BMTAP-7 with minimum inhibitory concentration (MIC) value between 4 and $8 \mu \mathrm{g} / \mathrm{mL}$ showed obvious bacteriostatic effect compared with that of TMTAP-7 and DTTAP-7 (Figures 5). In addition, the bacterial colony size of BMTAP-7 gradually decreased with the increase of concentrations (Figure 5A1 to 5C1).

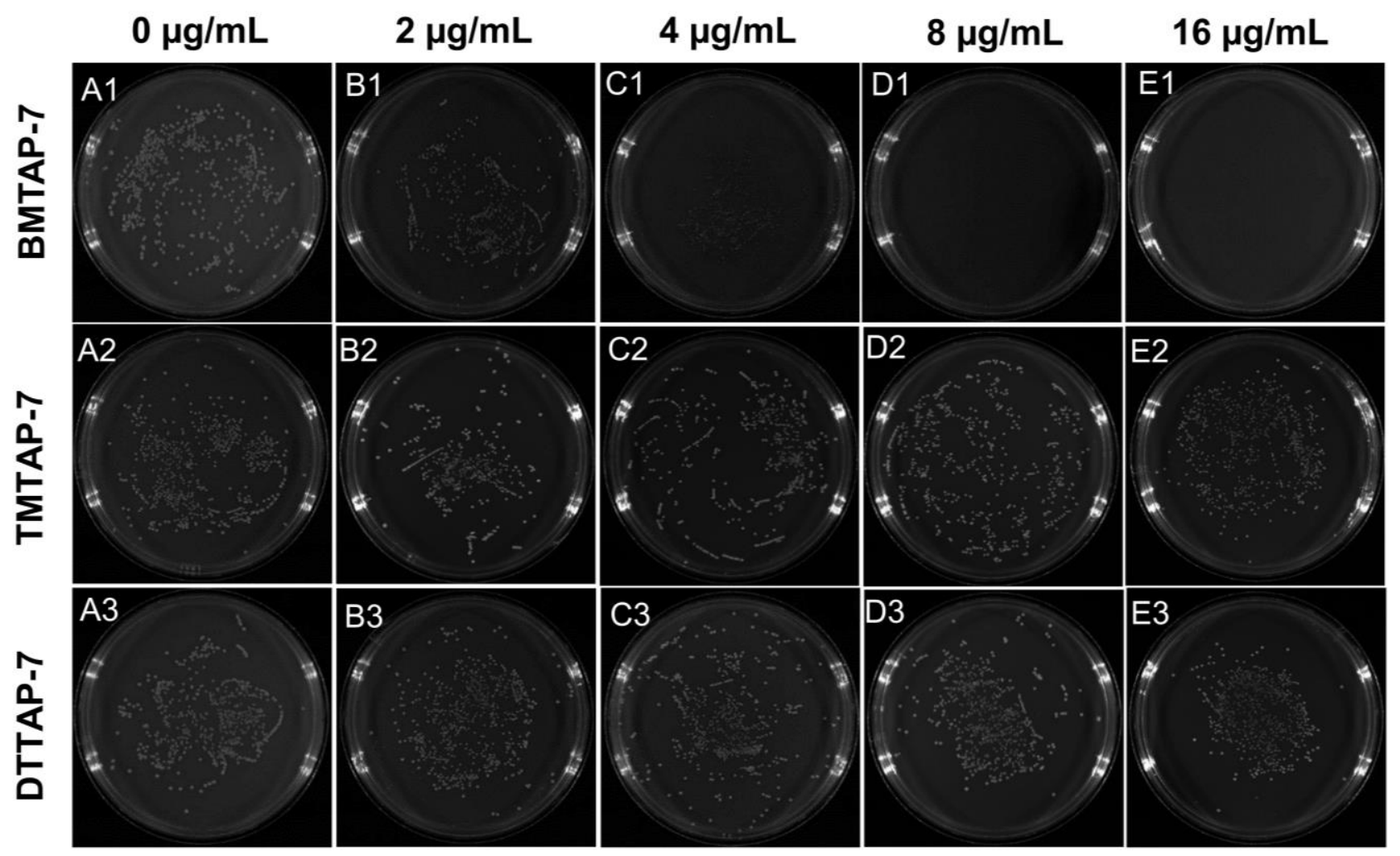

Figure 5. Bacterial inhibition tests of BMTAP-7, TMTAP-7 and DTTAP-7 in agar plates of different concentrations ranging from 0 $\mu \mathrm{g} / \mathrm{mL}$ to $16 \mu \mathrm{g} / \mathrm{mL}$ in petri dish. 

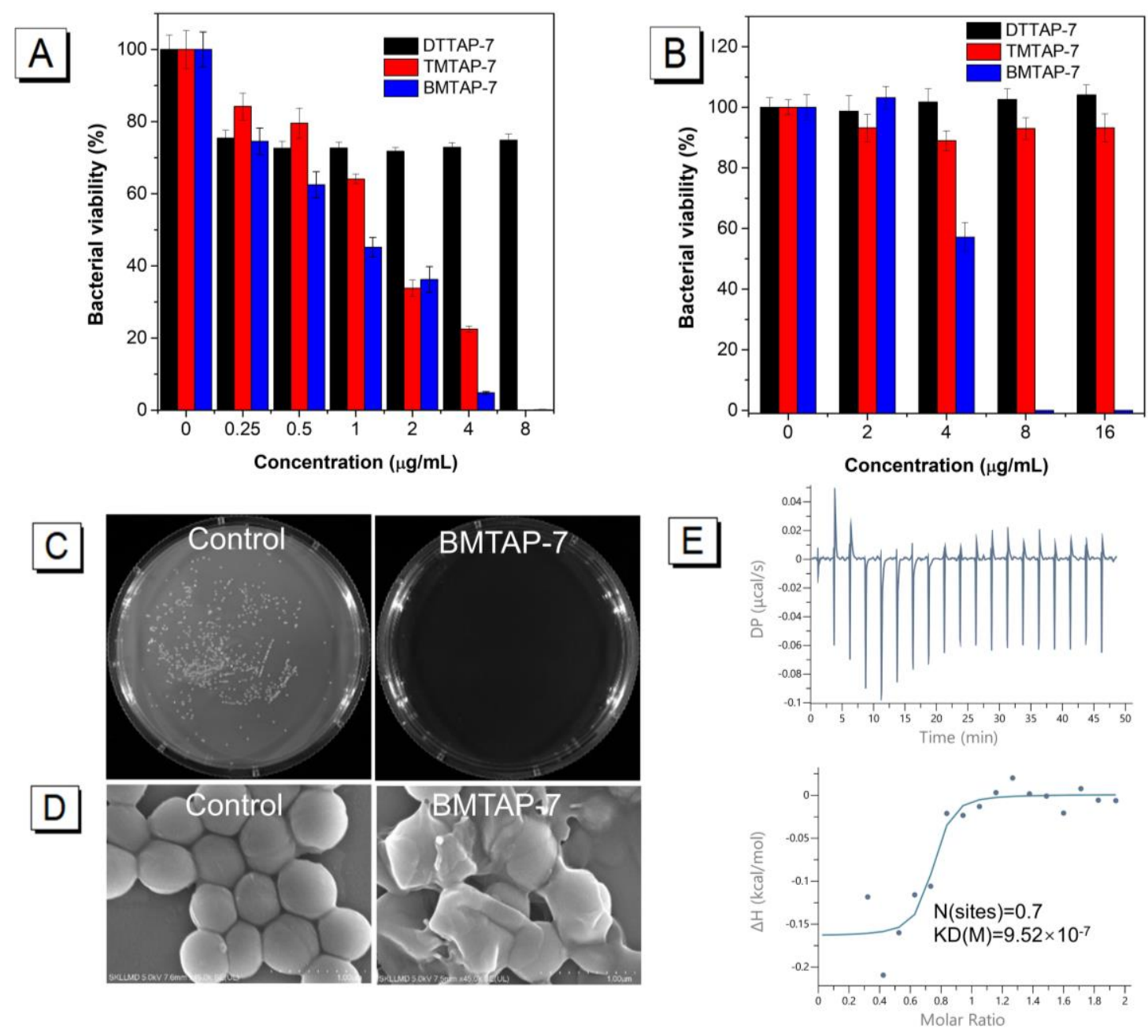

Figure 6. A) Bacteriostasis tests of three AIEgens against S. aureus in Nutrient Broth culture or B) agar plates of different AIEgens concentrations. C) Surface bacteriostatic experiments: BMTAP-7 and S. aureus were coated on the surface of the agar plates in turn. D) Morphological change of S. aureus for various treatments by SEM. E) ITC results of BMTAPC-7 with lipoteichoic acid (LTA). $[$ BMTAPC-7] $=2.0 \mathrm{mM}[\mathrm{LTA}]=0.2 \mathrm{mM}$

To more accurately reflect the bacteriostatic ability of BMTAP-7, TMTAP-7 and DTTAP-7, their MIC were tested in liquid medium. The MIC values of BMTAP-7 and TMTAP-7 are between 4 and $8 \mu \mathrm{g} / \mathrm{mL}$ (Figure 6A), while DTTAP-7 showed no significant antibacterial activity. Notably, TMTAP-7 with significant bacteriostatic activity in liquid medium showed little inhibitory effect in agar plates (Figure 6B), which might be caused by the poor hydrophilicity and non-uniform dispersion in agar plates. Moreover, to prove antibacterial ability of BMTAP-7 by surface contact, BMTAP-7 and $S$. aureus were coated on the surface of the agar medium in turn. As shown in Figure 6C, bacterial colonies become visible in the control group, but not in the group with BMTAP-7, revealing that it could inhibit reproduction through surface contact on $S$. aureus.

To explore the bacteriostatic mechanism of BMTAP-7 toward $S$. aureus, the morphological changes of bacteria with various treatments were studied by scanning electron microscope (SEM). After S. aureus were cultured with $8 \mu \mathrm{g} / \mathrm{mL}$ of BMTAP-7 in liquid medium for 24 hours, it was found that the outer membrane of bacteria became out of shape and folded, while the $S$. aureus in the control group kept a smooth outer membrane surface. Thus BMTAP-7 could destroy the outer membrane of bacteria to achieve bacteriostatic behavior (Figure 6D). Furthermore, considering that the activity of inhibitory reproduction by surface contact is related to the outer membrane surface structure, and lipoteichoic acid (LTA) is the main component in the outer membrane of $S$. aureus, the binding activity of BMTAPC-7 and LTA were studied by isothermal titration calorimetry (Figure 6E), which showed that their binding affinity is quite strong. Thus, it is concluded that BMTAP-7 could inhibit bacterial growth through electrostatic interaction with LTA of outer membrane on S. aureus in surface of medium.

\section{Conclusion}

A series of AIE-active cationic tricyclic 2-aminopyridinium derivatives were designed and synthesized via an efficient multi-component reaction. The investigation of the photophysical properties of BMTAPC-7 and BMTAPC-5 revealed that the RIV of seven-membered ring is the cause of the AIE effect. The increase in the hydrophobicity from BMTAP-7 to TMTAP-7 and to DTTAP-7 resulted in changes from mitochondria to lysosomes imaging in live cells, 
demonstrating good targeting toward organelles. Moreover, BMTAP-7, TMTAP-7 and DTTAP-7 can be used for specifically staining gram-positive bacteria, and BMTAP-7 could distinguish dead and live Gram-negative bacteria and fungi. The specific imaging is related to the structures and permeability of the outer membrane of Gram-negative bacteria and fungi. Furthermore, the MIC value of BMTAP-7 is between 4 and $8 \mu \mathrm{g} / \mathrm{mL}$ for $S$. aureus in liquid and solid medium, suggestive of excellent bacteriostatic ability. The SEM measurements indicate that the damage of outer membrane is one of the reasons to inhibit bacterial reproduction in liquid medium, and data of ITC indicated that LTA on outer membrane of $S$. aureus could be the target of bacterial inhibition through surface contact. Thus, this work demonstrates that the AIE-active tricyclic 2-aminopyridinium derivatives are a "one for all" system enjoys the multiple functions, such as organelle targeting, bacterial discrimination and inhibition in a single fluorescent molecule, which is of great significance to visualize the in-situ bacteriostatic process for bacteria in pharmacology.

\section{ASSOCIATED CONTENT}

Supporting Information. Synthesis and characterization, the spectral data, fluorescence images of cells and bacteria, HRMS, NMR spectra. This material is available free of charge via the Internet at http://pubs.acs.org.

\section{AUTHOR INFORMATION}

\section{Corresponding Author \\ *E-mail: msqinaj@scut.edu.cn (A. Qin) \\ *E-mail: tangbenz@ust.hk (B. Z. Tang)}

\section{Notes}

The authors declare no competing financial interest.

\section{ACKNOWLEDGMENT}

This work was financially supported by the National Natural Science Foundation of China (21788102, 21525417, and 51620105009), the Natural Science Foundation of Guangdong Province (2019B030301003, and 2016A030312002), the National Key Research and Development Program of China (Intergovernmental cooperation project, 2017YFE0132200) and the Innovation and Technology Commission of Hong Kong (ITC-CNERC14S01).

\section{REFERENCES}

(1) Wolfe, N. D.; Dunavan, C. P.; Diamond, J., Origins of major human infectious diseases. Nature 2007, 447, 279-283.

(2) Didelot, X.; Bowden, R.; Wilson, D. J.; Peto, T. E. A.; Crook, D. W., Transforming clinical microbiology with bacterial genome sequencing. Nat. Rev. Genet. 2012, 13, 601-612.

(3) Zhang, J.; Chen, Y. P.; Miller, K. P.; Ganewatta, M. S.; Bam, M.; Yan, Y.; Nagarkatti, M.; Decho, A. W.; Tang, C., Antimicrobial Metallopolymers and Their Bioconjugates with Conventional Antibiotics against Multidrug-Resistant Bacteria. J. Am. Chem. Soc. 2014, 136, 4873-4876.

(4) O'Connell, K. M. G.; Hodgkinson, J. T.; Sore, H. F.; Welch, M.; Salmond, G. P. C.; Spring, D. R., Combating Multidrug-Resistant Bacteria: Current Strategies for the Discovery of Novel Antibacterials. Angew. Chem. Int. Ed. 2013, 52, 10706-10733.
(5) Martinez-Garcia, M.; Swan, B. K.; Poulton, N. J.; Gomez, M. L.; Masland, D.; Sieracki, M. E.; Stepanauskas, R., High-throughput single-cell sequencing identifies photoheterotrophs and chemoautotrophs in freshwater bacterioplankton. ISME J. 2012, 6, 113-123.

(6) Bertozzi, C. R.; Bednarski, M. D. Antibody Targeting to Bacterial Cells Using Receptor-Specific Ligands. J. Am. Chem. Soc.1992, $114,2242-2245$.

(7) Clifford, R. J.; Milillo, M.; Prestwood, J.; Quintero, R.; Zurawski, D. V.; Kwak, Y. I.; Waterman, P. E.; Lesho, E. P.; Mc, G. P. Detection of Bacterial 16S rRNA and Identification of Four Clinically Important Bacteria by Real-Time PCR. PLoS One 2012, 7, e48558.

(8) Miranda, O. R.; Li, X.; Garcia-Gonzalez, L.; Zhu, Z. J.; Yan, B.; Bunz, U. H.; Rotello, V. M., Colorimetric Bacteria Eensing Using a Supramolecular Enzyme-Nanoparticle Biosensor. J. Am. Chem. Soc. 2011, 133, 9650-9653.

(9) Antaris, A. L.; Chen, H.; Cheng, K.; Sun, Y.; Hong, G.; Qu, C.; Diao, S.; Deng, Z.; Hu, X.; Zhang, B.; Zhang, X.; Yaghi, O. K.; Alamparambil, Z. R.; Hong, X.; Cheng, Z.; Dai, H. A Small-Molecule Dye for NIR-II Imaging. Nat. Mater. 2016, 15, 235-242.

(10) Duarte, A.; Chworos, A.; Flagan, S. F.; Hanrahan, G.; Bazan, G. C., Identification of Bacteria by Conjugated Oligoelectrolyte/SingleStranded DNA Electrostatic Complexes. J. Am. Chem. Soc. 2010, 132, 12562-12564.

(11) Giepmans, B. N.; Adams, S. R.; Ellisman, M. H.; Tsien, R. Y. The Fluorescent Toolbox for Assessing Protein Location and Function. Science 2006, 312, 217-224.

(12) Zhu, C.; Liu, L.; Yang, Q.; Lv, F.; Wang, S., Water-Soluble Conjugated Polymers for Imaging, Diagnosis, and Therapy. Chem. Rev. 2012, 112, 4687-4735.

(13) Yuan, H.; Liu, Z.; Liu, L.; Lv, F.; Wang, Y.; Wang, S., Cationic Conjugated Polymers for Discrimination of Microbial Pathogens. Adv. Mater. 2014, 26, 4333-4338.

(14) Pei, H.; Li, J.; Lv, M.; Wang, J.; Gao, J.; Lu, J.; Li, Y.; Huang, Q.; $\mathrm{Hu}$, J.; Fan, C., A Graphene-Based Sensor Array for High-Precision and Adaptive Target Identification with Ensemble Aptamers. J. Am. Chem. Soc. 2012, 134, 13843-13849.

(15) Chen, W.; Li, Q.; Zheng, W.; Hu, F.; Zhang, G.; Wang, Z.; Zhang, D.; Jiang, X., Identification of Bacteria in Water by a Fluorescent Array. Angew. Chem. Int. Ed. 2014, 53, 13734-13739.

(16) Gao, J.; Li, L.; Ho, P.-L.; Mak, G. C.; Gu, H.; Xu, B., Combining Fluorescent Probes and Biofunctional Magnetic Nanoparticles for Rapid Detection of Bacteria in Human Blood. Adv. Mater. 2006, 18, 3145-3148.

(17) Kwon, H.-Y.; Liu, X.; Choi, E. G.; Lee, J. Y.; Choi, S.-Y.; Kim, J.-Y.; Wang, L.; Park, S.-J.; Kim, B.; Lee, Y.-A.; Kim, J.-J.; Kang, N. Y.; Chang, Y.-T., Development of a Universal Fluorescent Probe for Gram-Positive Bacteria. Angew. Chem. Int. Ed. 2019, 58, 8426-8431.

(18) Chinen, A. B.; Guan, C. M.; Ferrer, J. R.; Barnaby, S. N.; Merkel, T. J.; Mirkin, C. A., Nanoparticle Probes for the Detection of Cancer Biomarkers, Cells, and Tissues by Fluorescence. Chem. Rev. 2015, 115, 10530-10574.

(19) Ding, D.; Li, K.; Liu, B.; Tang, B. Z., Bioprobes Based on AIE Fluorogens. Acc. Chem. Res. 2013, 46, 2441-2453.

(20) Liu, Z.; Zou, H.; Zhao, Z.; Zhang, P.; Shan, G.-G.; Kwok, R. T. K.; Lam, J. W. Y.; Zheng, L.; Tang, B. Z., Tuning Organelle Specificity and Photodynamic Therapy Efficiency by Molecular Function Design. ACS Nano 2019, 13, 11283-11293.

(21) Mei, J.; Leung, N. L. C.; Kwok, R. T. K.; Lam, J. W. Y.; Tang, B. Z., Aggregation-Induced Emission: Together We Shine, United We Soar! Chem. Rev. 2015, 115, 11718-11940.

(22) Mei, J.; Hong, Y.; Lam, J. W. Y.; Qin, A.; Tang, Y.; Tang, B. Z., Aggregation-Induced Emission: The Whole Is More Brilliant than the Parts. Adv. Mater. 2014, 26, 5429-5479.

(23) Xie, S.; Wong, A. Y. H.; Chen, S.; Tang, B. Z., Fluorogenic Detection and Characterization of Proteins by Aggregation-Induced Emission Methods. Chem. Eur. J. 2019, 25, 5824-5847.

(24) Zhao, E.; Chen, Y.; Wang, H.; Chen, S.; Lam, J. W. Y.; Leung, C. W. T.; Hong, Y.; Tang, B. Z., Light-Enhanced Bacterial Killing and 
Wash-Free Imaging Based on AIE Fluorogen. ACS App. Mater. Interfaces 2015, 7, 7180-7188.

(25) Gu, X.; Kwok, R. T. K.; Lam, J. W. Y.; Tang, B. Z., AIEgens for biological process monitoring and disease theranostics. Biomaterials 2017, 146, 115-135.

(26) Wan, Q.; Zhang, R.; Zhuang, Z.; Li, Y.; Huang, Y.; Wang, Z.; Zhang, W.; Hou, J.; Tang, B. Z., Molecular Engineering to Boost AIEActive Free Radical Photogenerators and Enable High-Performance Photodynamic Therapy under Hypoxia. Adv. Funct. Mater. 2020, n/a, 2002057.

(27) Li, Y.; Zhao, Z.; Zhang, J.; Kwok, R. T. K.; Xie, S.; Tang, R.; Jia, Y.; Yang, J.; Wang, L.; Lam, J. W. Y.; Zheng, W.; Jiang, X.; Tang, B. Z., A Bifunctional Aggregation-Induced Emission Luminogen for Monitoring and Killing of Multidrug-Resistant Bacteria. Adv. Funct. Mater. 2018, 28, 1804632.

(28) Zhou, T.; Hu, R.; Wang, L.; Qiu, Y.; Zhang, G.; Deng, Q.; Zhang, H.; Yin, P.; Situ, B.; Zhan, C.; Qin, A.; Tang, B. Z., An AIE-Active Conjugated Polymer with High ROS-Generation Ability and Biocompatibility for Efficient Photodynamic Therapy of Bacterial Infections. Angew. Chem. Int. Ed. 2020, 59, 9952-9956.

(29) Ge, X.; Gao, M.; Situ, B.; Feng, W.; He, B.; He, X.; Li, S.; Ou, Z.; Zhong, Y.; Lin, Y.; Ye, X.; Hu, X.; Tang, B. Z.; Zheng, L., One-step, rapid fluorescence sensing of fungal viability based on a bioprobe with aggregation-induced emission characteristics. Mater. Chem. Front. 2020, 4, 957-964.

(30) Wu, W.; Mao, D.; Xu, S.; Ji, S.; Hu, F.; Ding, D.; Kong, D.; Liu, B., High performance photosensitizers with aggregation-induced emission for image-guided photodynamic anticancer therapy. $\mathrm{Ma}$ ter. Horiz. 2017, 4, 1110-1114.

(31) Li, Y. J.; Zhang, H. T.; Chen, X. Y.; Gao, P. F.; Hu, C.-H., A multifunctional AIEgen with high cell-penetrating ability for intracellular fluorescence assays, imaging and drug delivery. Mater. Chem. Front. 2019, 3, 1151-1158.

(32) Chen, X.; Huang, L.; Jia, Y.; Hu, R.; Gao, M.; Ren, L.; Tang, B. Z., AIE-Based Theranostic Probe for Sequential Imaging and Killing of Bacteria and Cancer Cells. Adv. Opt. Mater. 2020, 8, 1902191.

(33) Jiao, Z.; Guo, Z.; Huang, X.; Fan, H.; Zhao, M.; Zhou, D.; Ruan, X.; Zhang, P.; Zhou, S.; Tang, B. Z., On-site visual discrimination of transgenic food by water-soluble DNA-binding AIEgens. Mater. Chem. Front. 2019, 3, 2647-2651.

(34) Wang, D.; Lee, M. M. S.; Shan, G.; Kwok, R. T. K.; Lam, J. W. Y.; Su, H.; Cai, Y.; Tang, B. Z., Highly Efficient Photosensitizers with FarRed/Near-Infrared Aggregation-Induced Emission for In Vitro and In Vivo Cancer Theranostics. Adv. Mater. 2018, 30, 1802105.

(35) Guo, D.; Li, L.; Zhu, X.; Heeney, M.; Li, J.; Dong, L.; Yu, Q.; Gan, Z.; Gu, X.; Tan, L., Naphthalene diimide based near-infrared luminogens with aggregation-induced emission characteristics for biological imaging and high mobility ambipolar transistors. Sci. China Chem. 2020, 63, 1198-1207.

(36) Zhu, C.; Kwok, R. T. K.; Lam, J. W. Y.; Tang, B. Z., AggregationInduced Emission: A Trailblazing Journey to the Field of Biomedicine. ACS Appl. Bio Mater. 2018, 1, 1768-1786.

(37) Liu, X.; Li, M.; Han, T.; Cao, B.; Qiu, Z.; Li, Y.; Li, Q.; Hu, Y.; Liu, Z.; Lam, J. W. Y.; Hu, X.; Tang, B. Z., In Situ Generation of AzoniaContaining Polyelectrolytes for Luminescent Photopatterning and Superbug Killing. J. Am. Chem. Soc. 2019, 141, 11259-11268.

(38) Liu, B.; He, W.; Lu, H.; Wang, K.; Huang, M.; Kwok, R. T. K.; Lam, J. W. Y.; Gao, L.; Yang, J.; Tang, B., A facile design for multifunctional AIEgen based on tetraaniline derivatives. Sci. China Chem. 2019, 62, 732-738.
(39) Zhang, T.; Li, Y.; Zheng, Z.; Ye, R.; Zhang, Y.; Kwok, R. T. K.; Lam, J. W. Y.; Tang, B. Z., In Situ Monitoring Apoptosis Process by a Self-Reporting Photosensitizer. J. Am. Chem. Soc. 2019, 141, 56125616.

(40) Niu, G.; Zhang, R.; Gu, Y.; Wang, J.; Ma, C.; Kwok, R. T. K.; Lam, J. W. Y.; Sung, H. H. Y.; Williams, I. D.; Wong, K. S.; Yu, X.; Tang, B. Z., Highly photostable two-photon NIR AIEgens with tunable organelle specificity and deep tissue penetration. Biomaterials 2019, 208, 72-82.

(41) Zhou, C.; Xu, W.; Zhang, P.; Jiang, M.; Chen, Y.; Kwok, R. T. K.; Lee, M. M. S.; Shan, G.; Qi, R.; Zhou, X.; Lam, J. W. Y.; Wang, S.; Tang, B. Z., Engineering Sensor Arrays Using Aggregation-Induced Emission Luminogens for Pathogen Identification. Adv. Funct. Mater. 2019, 29, 1805986

(42) Hu, Q.; Gao, M.; Feng, G.; Liu, B., Mitochondria-Targeted Cancer Therapy Using a Light-Up Probe with Aggregation-InducedEmission Characteristics. Angew. Chem. Int. Ed. 2014, 53, 1422514229.

(43) Wang, D.; Su, H.; Kwok, R. T. K.; Hu, X.; Zou, H.; Luo, Q.; Lee, Michelle M. S.; Xu, W.; Lam, J. W. Y.; Tang, B. Z., Rational design of a water-soluble NIR AIEgen, and its application in ultrafast washfree cellular imaging and photodynamic cancer cell ablation. Chem. Sci. 2018, 9, 3685-3693.

(44) Kang, M.; Zhou, C.; Wu, S.; Yu, B.; Zhang, Z.; Song, N.; Lee, M. M. S.; Xu, W.; Xu, F.-J.; Wang, D.; Wang, L.; Tang, B. Z., Evaluation of Structure-Function Relationships of Aggregation-Induced Emission Luminogens for Simultaneous Dual Applications of Specific Discrimination and Efficient Photodynamic Killing of Gram-Positive Bacteria. J. Am. Chem. Soc. 2019, 141, 16781-16789.

(45) Hu, R.; Zhou, F.; Zhou, T.; Shen, J.; Wang, Z.; Zhao, Z.; Qin, A.; Tang, B. Z., Specific discrimination of gram-positive bacteria and direct visualization of its infection towards mammalian cells by a DPAN-based AIEgen. Biomaterials 2018, 187, 47-54.

(46) Bu, F.; Duan, R.; Xie, Y.; Yi, Y.; Peng, Q.; Hu, R.; Qin, A.; Zhao, Z.; Tang, B. Z., Unusual Aggregation-Induced Emission of a Coumarin Derivative as a Result of the Restriction of an Intramolecular Twisting Motion. Angew. Chem. Int. Ed. 2015, 54, 14492-14497.

(47) Bakulina, O.; Merkt, F. K.; Knedel, T.-O.; Janiak, C.; Müller, T. J. J., Synthesis of Water-Soluble Blue-Emissive Tricyclic 2-Aminopyridinium Salts by Three-Component Coupling-(3+3)-Anellation. Angew. Chem. Int. Ed. 2018, 57, 17240-17244.

(48) Lee, M. M. S.; Xu, W.; Zheng, L.; Yu, B.; Leung, A. C. S.; Kwok, R. T. K.; Lam, J. W. Y.; Xu, F.-J.; Wang, D.; Tang, B. Z., Ultrafast discrimination of Gram-positive bacteria and highly efficient photodynamic antibacterial therapy using near-infrared photosensitizer with aggregation-induced emission characteristics. Biomaterials 2020, 230, 119582.

(49) Huang, H.; Yang, L.; Zhang, P.; Qiu, K.; Huang, J.; Chen, Y.; Diao, J.; Liu, J.; Ji, L.; Long, J.; Chao, H., Real-time tracking mitochondrial dynamic remodeling with two-photon phosphorescent iridium (III) complexes. Biomaterials 2016, 83, 321-331.

(50) Zielonka, J.; Joseph, J.; Sikora, A.; Hardy, M.; Ouari, O.; Vasquez-Vivar, J.; Cheng, G.; Lopez, M.; Kalyanaraman, B., Mitochondria-Targeted Triphenylphosphonium-Based Compounds: Syntheses, Mechanisms of Action, and Therapeutic and Diagnostic Applications. Chem. Rev. 2017, 117, 10043-10120. 
Table of Content

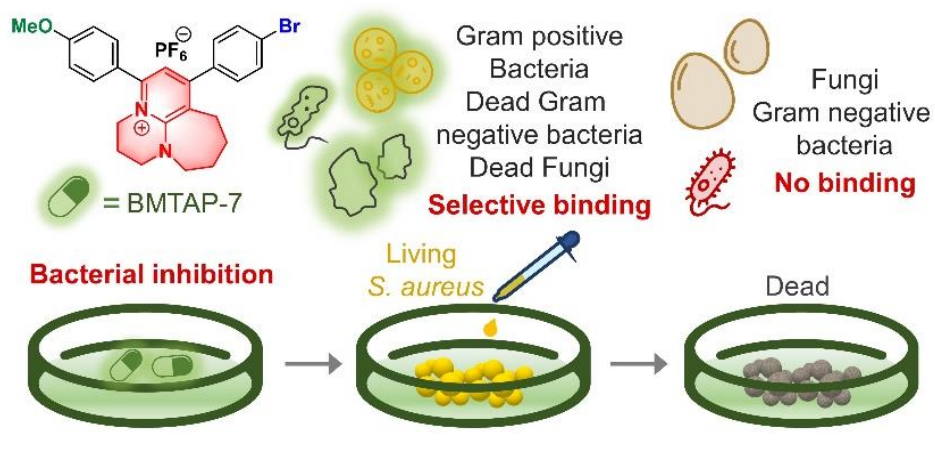

9 